\title{
GEOMETRIC ERGODICITY AND QUASI-STATIONARITY IN DISCRETE-TIME BIRTH-DEATH PROCESSES
}

\author{
ERIK A. VAN DOORN and PAULINE SCHRIJNER ${ }^{1}$
}

(Received 22 September 1993)

\begin{abstract}
We study two aspects of discrete-time birth-death processes, the common feature of which is the central role played by the decay parameter of the process. First, conditions for geometric ergodicity and bounds for the decay parameter are obtained. Then the existence and structure of quasi-stationary distributions are discussed. The analyses are based on the spectral representation for the $n$-step transition probabilities of a birth-death process developed by Karlin and McGregor.
\end{abstract}

\section{Introduction}

Throughout we use the term random walk for a discrete-time birth-death process $X \equiv(X(n), n=0,1, \ldots)$, on the state space $\{0,1, \ldots\}$ and with stationary 1-step transition probabilities $P_{i j} \equiv \operatorname{Pr}\{X(n+1)=j \mid X(n)=i\}$. We write $p_{j} \equiv P_{j, j+1}$, $q_{j+1} \equiv P_{j+1, j}$ and $r_{j} \equiv P_{j j}$, so that

$$
P \equiv\left(P_{i j}\right)=\left[\begin{array}{ccccccc}
r_{0} & p_{0} & 0 & 0 & . & . & \cdot \\
q_{1} & r_{1} & p_{1} & 0 & . & . & . \\
0 & q_{2} & r_{2} & p_{2} & . & . & . \\
. & \cdot & \cdot & \cdot & . & . & . \\
. & . & . & . & . & . & \cdot
\end{array}\right]
$$

and assume $p_{j}>0, q_{j+1}>0$ and $r_{j} \geq 0$. In addition, we require $p_{j}+q_{j}+r_{j}=1$ for $j \geq 1$, but we allow $q_{0} \equiv 1-p_{0}-r_{0} \geq 0$. If $q_{0}=0$, then $P$ is stochastic and the random walk $X$ is said to have a reflecting barrier 0 . If $q_{0}>0$ then $P$ is strictly substochastic and the random walk has an ignored absorbing state which can be reached through state 0 only. The absorbing state, if present, will be designated as -1 .

\footnotetext{
'Faculty of Applied Math., University of Twente, 7500 AE Enschede, The Netherlands.
}

(C) Australian Mathematical Society, 1995, Serial-fee code 0334-2700/95 
In what follows we discuss some aspects of random walks, the common feature of which is the dominant role played by a quantity called the decay parameter. The decay parameter $\gamma(X)$ of a random walk $X$ is a number in the interval $(0,1]$ which characterizes the speed with which the $n$-step transition probabilities of $X$ go to their limiting values as $n \rightarrow \infty$. If $\gamma(X)<1$, then these probabilities go to their limits geometrically fast and $X$ is called geometrically ergodic. In Section 3 , we obtain bounds for the decay parameter and conditions for geometric ergodicity of a random walk.

A quasi-stationary distribution of a random walk $X$ with an absorbing state -1 is an initial distribution such that the conditional probability of the random walk being in state $j$ at time $n$, given that no absorption has occurred by that time, is independent of $n$ for all $j$. We prove in Section 4 that quasi-stationary distributions for $X$ exist if and only if absorption at -1 is certain and $X$ is geometrically ergodic. In addition, we obtain expressions for these quasi-stationary distributions. Our results generalize and supplement the recent findings of Ferrari, Martínez and Picco [11].

Our method of analysis is linked with the spectral representation of the $n$-step transition probabilities of a random walk, developed by Karlin and McGregor [15]. In Section 2, we introduce this spectral representation and establish some ramifications which are pertinent to our purposes, but also of independent interest. In particular, we present some properties of the sequence of orthogonal polynomials which is associated with a random walk, and of the orthogonalizing measure, and show how properties of a random walk such as transience and recurrence are reflected by this measure. We conclude in Section 5 with some examples.

The present paper constitutes to some extent the discrete-time counterpart of van Doorn [5] and van Doorn [7], which discuss exponential ergodicity and quasistationarity, respectively, in continuous-time birth-death processes. However, a study of limiting conditional (or quasi-limiting) distributions as in [7], which turned out to be considerably more complicated in discrete time, has been relegated to a separate paper, see van Doorn and Schrijner [9].

Quasi-stationary distributions for continuous-time Markov chains have recently attracted much attention because of their theoretical and practical interest, see for example, Ferrari, Kesten, Martínez and Picco [10], Kijima [20], Nair and Pollett [22], Pollett [24], and the references in these papers. In retrospect, one can say that in this continuous-time setting several results for birth-death processes were indicative of results that hold more generally. One of the motives for the present study is the expectation that the same will be true in discrete time. 


\section{Preliminaries}

2.1. Basic properties Let $X \equiv(X(n), n=0,1, \ldots)$ be a random walk as defined in Section 1. It is clear that the state space $\{0,1, \ldots\}$ of $X$ constitutes an irreducible class which is aperiodic if $r_{j}>0$ for some $j$, and periodic with period 2 if $r_{j}=0$ for all $j$. Accordingly, $X$ will be called aperiodic if $r_{j}>0$ for some $j$ and periodic if $r_{j}=0$ for all $j$.

We define

$$
\pi_{0} \equiv 1 ; \quad \pi_{j} \equiv \frac{p_{0} p_{1} \ldots p_{j-1}}{q_{1} q_{2} \ldots q_{j}}, j \geq 1,
$$

and, noting that $\sum_{j}\left(\pi_{j}+\left(p_{j} \pi_{j}\right)^{-1}\right)=\infty$, quote the following result from, for example, Karlin and McGregor [15].

THEOREM 2.1. If $q_{0}=0$, then the random walk $X$ is

$$
\text { recurrent } \Leftrightarrow \sum_{j=0}^{\infty}\left(p_{j} \pi_{j}\right)^{-1}=\infty
$$

and

$$
\text { positive recurrent } \Leftrightarrow \sum_{j=0}^{\infty} \pi_{j}<\infty
$$

When $q_{0}>0$, there is a positive probability of absorption at -1 , so that the random walk is transient. Absorption at -1 is called certain if the probability of eventual absorption equals 1 , and is called ergodic if it is certain and the expected time to absorption is finite. Absorption is called transient if it is not certain. These properties are independent of the initial state, and using the previous theorem we readily obtain the following criteria (see Harris [12]).

THEOREM 2.2. If $q_{0}>0$, then absorption at -1 is

$$
\text { certain } \Leftrightarrow \sum_{j=0}^{\infty}\left(p_{j} \pi_{j}\right)^{-1}=\infty
$$

and

$$
\text { ergodic } \Leftrightarrow \sum_{j=0}^{\infty} \pi_{j}<\infty
$$

We write $p_{i j}(n) \equiv \operatorname{Pr}\{X(n)=j \mid X(0)=i\}$ for the $n$-step transition probabilities, and note that they tend to zero as $n \rightarrow \infty$ unless $X$ is positive recurrent (and hence $q_{0}=0$ ). In the latter case,

$$
\lim _{n \rightarrow \infty} \frac{1}{n} \sum_{k=1}^{n} p_{i j}(k)=\pi_{j}\left(\sum_{k=0}^{\infty} \pi_{k}\right)^{-1},
$$


and so

$$
X \text { is aperiodic } \Rightarrow \lim _{n \rightarrow \infty} p_{i j}(n)=\pi_{j}\left(\sum_{k=0}^{\infty} \pi_{k}\right)^{-1},
$$

but interpreting $\left(\sum_{k} \pi_{k}\right)^{-1}$ as zero if $\sum_{k} \pi_{k}=\infty,(2.2)$ and (2.3) are valid whether $X$ is positive recurrent or not.

2.2. The spectral representation Karlin and McGregor [15] have shown that the transition probabilities $p_{i j}(n)$ can be represented as

$$
p_{i j}(n)=\pi_{j} \int_{-1}^{1} x^{n} Q_{i}(x) Q_{j}(x) d \psi(x) .
$$

Here $\pi_{j}, j \geq 0$, are the constants defined in (2.1) and $\left\{Q_{j}(x)\right\}_{j=0}^{\infty}$ is a sequence of polynomials defined by the recurrence relations

$$
\begin{aligned}
& Q_{0}(x)=1, \quad p_{0} Q_{1}(x)=x-r_{0} \\
& x Q_{j}(x)=q_{j} Q_{j-1}(x)+r_{j} Q_{j}(x)+p_{j} Q_{j+1}(x), j \geq 1 .
\end{aligned}
$$

Finally, $\psi$ is the (unique) measure of total mass 1 and infinite support in the interval $[-1,1]$ with respect to which the polynomials $\left\{Q_{j}(x)\right\}_{j}$ are orthogonal; indeed, substituting $n=0$ in (2.4) yields

$$
\pi_{j} \int_{-1}^{1} Q_{i}(x) Q_{j}(x) d \psi(x)=\delta_{i j}
$$

In what follows any sequence of polynomials defined by a recurrence relation of the type (2.5), where $p_{j}>0, q_{j+1}>0$ and $r_{j} \geq 0$ for $j \geq 0, q_{0} \equiv 1-p_{0}-r_{0} \geq 0$ and $p_{j}+q_{j}+r_{j}=1$ for $j \geq 1$, will be called a sequence of random walk polynomials; the corresponding measure will be called a random walk measure. Because of the crucial role random walk polynomials play in our analysis, we shall collect a number of pertinent properties of polynomials of this type, and of the measures that go with them.

2.3. Random walk polynomials Let $\left\{Q_{j}(x)\right\}_{j}$ be a sequence of random walk polynomials and $q_{0} \equiv 1-p_{0}-r_{0}$. Reference to the literature on orthogonal polynomials is sometimes facilitated by normalizing random walk polynomials such that they are monic. Thus we define $P_{0}(x) \equiv Q_{0}(x)=1$, and

$$
P_{j}(x) \equiv p_{0} p_{1} \ldots p_{j-1} Q_{j}(x), \quad j \geq 1,
$$

and note that the sequence $\left\{P_{j}(x)\right\}_{j}$ satisfies the recurrence relations

$$
\begin{aligned}
& P_{0}(x)=1, \quad P_{1}(x)=x-r_{0}, \\
& P_{j+1}(x)=\left(x-r_{j}\right) P_{j}(x)-p_{j-1} q_{j} P_{j-1}(x), j \geq 1 .
\end{aligned}
$$


From, for example, Chihara's [1] book on orthogonal polynomials we now see that $P_{j}(x)$, and hence $Q_{j}(x)$, has $j$ real distinct zeros $x_{j 1}<x_{j 2}<\ldots<x_{j j}$. These zeros have the separation property

$$
x_{j+1, i}<x_{j i}<x_{j+1, i+1}, \quad i=1,2, \ldots, j, j \geq 1,
$$

whence

$$
\xi_{i} \equiv \lim _{k \rightarrow \infty} x_{k i} \quad \text { and } \quad \eta_{j} \equiv \lim _{k \rightarrow \infty} x_{k, k-j+1}, \quad i, j \geq 1,
$$

exist in the extended real number system; also

$$
-\infty \leq \xi_{i} \leq \xi_{i+1}<\eta_{j+1} \leq \eta_{j} \leq \infty, \quad i, j \geq 1 .
$$

Actually, the $\xi_{i}$ 's and $\eta_{j}$ 's are bounded, as appears from the following lemma. In this lemma, as well as in what follows, we shall consistently write $\xi$ and $\eta$ instead of $\xi_{1}$ and $\eta_{1}$, respectively.

LEMMA 2.1. $-1 \leq \inf _{j}\left\{2 r_{j}-1\right\} \leq \xi<\eta \leq 1$.

Proof. Applying Theorem 2 in van Doorn [6] to the polynomials $P_{j}(x)$ of (2.8), and choosing $\chi_{j}=p_{j-2}, j \geq 2$, one obtains $\xi \geq \inf \left\{2 r_{j}-1\right\} \geq-1$. Similarly, the analogue for $\eta$ of the above theorem leads to $\eta \leq \sup \left\{p_{j}+q_{j}+r_{j}\right\}=1$.

We remark that any sequence of polynomials which is orthogonal with respect to a measure on $[-1,1]$ can be normalized to satisfy a recurrence relation of the type (2.5) (or (2.8)), where $p_{j}>0, q_{j+1}>0$. It is the fact $r_{j} \geq 0$ for all $j$ which distinguishes random walk polynomial sequences from the others (see van Doorn and Schrijner [8]). Some further properties of random walk polynomials are given in Appendix 1.

2.4. Random walk measures As noted in Subsection 2.2, a sequence of random walk polynomials $\left\{Q_{j}(x)\right\}_{j}$ is an orthogonal polynomial sequence with respect to a unique measure $\psi$ with infinite support

$$
\operatorname{supp}(\psi) \equiv\{x \mid \psi((x-\epsilon, x+\epsilon))>0 \text { for all } \epsilon>0\}
$$

in the interval $[-1,1]$ and total mass 1 . There is a close relation between supp $(\psi)$ and the quantities $\xi_{i}$ and $\eta_{j}$ defined in (2.10). Indeed, from (2.11) and Lemma 2.1 we conclude that both

$$
\sigma \equiv \lim _{i \rightarrow \infty} \xi_{i} \quad \text { and } \quad \tau \equiv \lim _{j \rightarrow \infty} \eta_{j}
$$

exist and $-1 \leq \sigma \leq \tau \leq 1$. Furthermore, Chihara [1, Theorem II.4.6] tells us

$$
\xi_{i+1}=\xi_{i} \Rightarrow \sigma=\xi_{i}, \quad i \geq 1,
$$


and

$$
\eta_{j+1}=\eta_{j} \Rightarrow \tau=\eta_{j}, \quad j \geq 1 .
$$

Now defining the (possibly finite) sets

$$
\Xi \equiv\left\{\xi \equiv \xi_{1}, \xi_{2}, \cdots\right\} \text { and } H \equiv\left\{\eta \equiv \eta_{1}, \eta_{2}, \cdots\right\}
$$

we obtain from Chihara [1, pages 61-63] the following lemma.

LEMMA 2.2. One has supp $(\psi)=\bar{\Xi} \cup S_{\ell} \cup \bar{H}$ (a bar denoting closure), where $S_{\ell}$ is a subset of $(\sigma, \tau)$; also, $\sigma$ is the smallest and $\tau$ is the largest limit point of $\operatorname{supp}(\psi)$.

It follows in particular that

$$
\xi=\inf \operatorname{supp}(\psi) \text { and } \eta=\sup \operatorname{supp}(\psi) .
$$

Thus, in view of Lemma 2.1, we now have verified that supp $(\psi) \subset[-1,1]$. We also note that if $\Xi$ and $H$ are finite, or, equivalently, if the phenomena described in (2.13) and (2.14) occur, then the set $S_{\ell}$ must contain infinitely many points, since supp ( $\psi$ ) is always infinite. If $\Xi$ or $H$ are infinite, then $S_{\ell}$ may be finite or even empty.

More precise information on supp $(\psi)$ may be obtained by looking at bounds for $\xi+\eta$ and $\sigma+\tau$. In particular, Theorem 13 in van Doorn [4] gives us

$$
2 \inf _{j}\left\{r_{j}\right\} \leq \xi+\eta \leq 2 \sup _{j}\left\{r_{j}\right\}
$$

but we can do slightly better as follows.

LEMMA 2.3. $\inf _{j}\left\{r_{j}+r_{j+1}\right\} \leq \xi+\eta \leq \sup _{j}\left\{r_{j}+r_{j+1}\right\}$.

PROOF. The proof parallels that of Theorem 13 in [4], except that, instead of (29) in that paper, we must now use (32) and choose $\beta_{j} \equiv\left(1-g_{j-1}\right) g_{j}$ with $g_{j} \equiv \gamma_{2 j} /\left(\gamma_{2 j}+\gamma_{2 j+1}\right)$.

In the same manner, Theorem 14 in [4] can be improved to yield the next lemma.

LEMMA 2.4. $\lim _{j \rightarrow \infty} \inf \left\{r_{j}+r_{j+1}\right\} \leq \sigma+\tau \leq \lim _{j \rightarrow \infty} \sup \left\{r_{j}+r_{j+1}\right\}$.

It follows in particular that

$$
\xi+\eta \geq 0 \text { and } \sigma+\tau \geq 0,
$$

that is, the smallest (limit) point of supp ( $\psi)$ is at least as close to 0 as the largest (limit) point of $\operatorname{supp}(\psi)$. This result can also be conceived as a consequence of Lemma 2 in Kent and Longford [17]. The following sufficient condition for strict inequality to hold in (2.16), partly noted earlier by Karlin and McGregor [15], can also be obtained from the Lemmas 2.3 and 2.4 . 
COROLLARY 2.1. If $r_{j} \geq \delta>0$ for all $j$, then $\xi+\eta \geq 2 \delta>0$ and $\sigma+\tau \geq 2 \delta>0$.

On the other hand, the Lemmas 2.3 and 2.4 imply that $\xi+\eta=\sigma+\tau=0$ if every $r_{j}$ is zero, that is, if the associated random walk is periodic. Actually, a much stronger result is valid, for it is easy to show (see [15]) that the random walk measure $\psi$ is symmetric about $x=0$ if and only if $r_{j}=0$ for all $j$. For this reason Karlin and McGregor [15] use the adjectives symmetric and asymmetric instead of periodic and aperiodic, respectively, to characterize a random walk.

Whitehurst [29, Theorem 5.2] has proved the interesting result

$$
X \text { is aperiodic } \Rightarrow \int_{-1}^{1}(\eta+x)^{-1} d \psi(x)<\infty .
$$

As a corollary, we note

$$
X \text { is aperiodic } \Rightarrow \psi(\{-\eta\})=0,
$$

that is, $\psi$ has no point mass at $-\eta$ if $r_{j}>0$ for some $j$. (In view of (2.15) and (2.16) this result is of course trivial if $\xi+\eta>0$.) Statement (2.18) generalizes a result of Karlin and McGregor's [15], which showed that the measure of an aperiodic random walk has no atom at -1 , but is a special case of a result of Kersting [18]. The result may seem surprising in view of the fact that when $X$ is periodic, that is, $\psi$ is symmetric, then an atom at $x=\eta$ is matched by an equal atom at $x=-\eta$.

Another consequence of (2.17), or rather (2.18), is

$$
X \text { is aperiodic and } \xi=-\eta \Rightarrow \sigma=\xi=-\eta=-\tau .
$$

Indeed, if $\xi=-\eta$ and $X$ is aperiodic, then, by $(2.18), \psi(\{\xi\})=0$, so that by Lemma 2.2, $\sigma=\xi$. Hence, by (2.16), $\tau \geq-\sigma=\eta$, so that $\tau=\eta$.

Further information on the random walk measure $\psi$ can be obtained by transforming the sequence of random walk polynomials into a sequence of birth-death polynomials, as suggested by Karlin and McGregor [15]. In this way many results from the theory of birth-death processes in continuous time (see Karlin and McGregor [13], [14] and van Doorn [5], [7]) can be translated in terms of random walks. Pursuing this approach we define

$$
R_{j}(x) \equiv Q_{j}(1-x), \quad j \geq 0,
$$

and

$$
\vartheta([0, x]) \equiv \psi([1-x, 1]), \quad 0 \leq x \leq 2 .
$$

It is easy to see that

$$
\begin{aligned}
& R_{0}(x)=1, \quad p_{0} R_{1}(x)=p_{0}+q_{0}-x \\
& -x R_{j}(x)=q_{j} R_{j-1}(x)-\left(p_{j}+q_{j}\right) R_{j}(x)+p_{j} R_{j+i}(x), j>1,
\end{aligned}
$$


where $q_{0} \equiv 1-p_{0}-r_{0}$, and

$$
\pi_{j} \int_{0}^{2} R_{i}(x) R_{j}(x) d \vartheta(x)=\delta_{i j}, \quad i, j \geq 0 .
$$

So $\left\{R_{j}(x)\right\}_{j}$ is a sequence of birth-death polynomials with birth rates $p_{j}$ and death rates $q_{j}$, and $\vartheta$ is the unique measure with respect to which the sequence $\left\{R_{j}(x)\right\}_{j}$ is orthogonal. Using the transformation (2.20), Lemma 6 (on page 527) of Karlin and McGregor [13] is readily seen to imply

$$
q_{0}=0 \Rightarrow \int_{-1}^{1}(1-x)^{-1} d \psi(x)=\sum_{j=0}^{\infty}\left(p_{j} \pi_{j}\right)^{-1}
$$

and

$$
q_{0}>0 \Rightarrow q_{0} \int_{-1}^{1}(1-x)^{-1} d \psi(x)=1-\lim _{j \rightarrow \infty}\left(Q_{j}(1)\right)^{-1} .
$$

Also, (9.19) of Karlin and McGregor [14] translates into

$$
q_{0}>0 \text { and } \sum_{j=0}^{\infty}\left(p_{j} \pi_{j}\right)^{-1}=\infty \Rightarrow q_{0}^{2} \int_{-1}^{1}(1-x)^{-2} d \psi(x)=\sum_{j=0}^{\infty} \pi_{j} .
$$

With this information the classification in the Theorems 2.1 and 2.2 can now be expressed in terms of $q_{0}$ and the random walk measure $\psi$ as follows.

THEOREM 2.3. (i) If $q_{0}=0$, then the random walk is

$$
\text { recurrent } \Leftrightarrow \int_{-1}^{1}(1-x)^{-1} d \psi(x)=\infty
$$

and

$$
\text { positive recurrent } \Leftrightarrow \psi(\{1\})>0 .
$$

(ii) If $q_{0}>0$, then the random walk is transient and absorption at -1 is

$$
\text { certain } \Leftrightarrow q_{0} \int_{-1}^{1}(1-x)^{-1} d \psi(x)=1
$$

and

$$
\text { ergodic } \Leftrightarrow q_{0} \int_{-1}^{1}(1-x)^{-1} d \psi(x)=1 \text { and } \int_{-1}^{1}(1-x)^{-2} d \psi(x)<\infty .
$$


PROOF. From Karlin and McGregor [15] (or from Corollary 2.6 of Shohat and Tamarkin [27]) we know

$$
\psi(\{1\})=\left\{\sum_{j=0}^{\infty} \pi_{j} Q_{j}^{2}(1)\right\}^{-1},
$$

which should be interpreted as zero if the series diverges. Also, the recurrence relation (2.5) yields

$$
Q_{j}(1)=1+q_{0} \sum_{k=0}^{j-1}\left(p_{k} \pi_{k}\right)^{-1}, \quad j \geq 0 .
$$

The first part of the theorem now follows from Theorem 2.1 and (2.24), while the second part is implied by Theorem 2.2, (2.25) and (2.26).

Theorem 2.3 allows us to formulate sufficient conditions for $\eta(=\sup \operatorname{supp}(\psi))$ to be equal to one as follows. (The reverses of these statements do not necessarily hold true.)

COROLlaRY 2.2. (i) If $q_{0}=0$ and the random walk is recurrent, then $\eta=1$. (ii) If $q_{0}>0$ and absorption at -1 is certain but not ergodic, then $\eta=1$.

\section{Geometric ergodicity}

3.1. Introduction In this section (with the exception of Subsection 3.5) we shall assume that the random walk $X$ is aperiodic. As a consequence, the limits

$$
p_{i j}=\lim _{n \rightarrow \infty} p_{i j}(n), \quad i, j \geq 0,
$$

exist and are independent of $i$.

It is of interest to characterize the speed with which the transition probabilities $p_{i j}(n)$ tend to their limiting values $p_{i j}$. To this end we define

$$
\gamma_{i j} \equiv \inf \left\{\rho \geq 0 \mid p_{i j}(n)-p_{i j}=\mathrm{O}\left(\rho^{n}\right) \text { as } n \rightarrow \infty\right\}
$$

and

$$
\gamma \equiv \sup \left\{\gamma_{i j}\right\}
$$

and call $\gamma$ the decay parameter of $X$. When $\gamma<1$ the random walk is said to be geometrically ergodic.

Several authors (Kendall [16], Vere-Jones [28], Kingman [21]) have studied geometric ergodicity in the framework of denumerable discrete-time Markov chains. Their main results, formulated in terms of the random walk $X$, may be summarized as follows. 
1. If $X$ is null-recurrent, then $\gamma_{i j}=1$ for all $i, j$, so that $\gamma=1$ and $X$ is not geometrically ergodic.

2. If $X$ is positive recurrent, then $\gamma<1$ if $\gamma_{i i}<1$ for some $i$. It may happen that $\gamma_{i j}<\gamma$ for some pairs $(i, j)$.

3. If $X$ is transient, then

$$
\lim _{n \rightarrow \infty}\left(p_{i j}(n)\right)^{1 / n}=\gamma, \quad i, j \geq 0,
$$

so that $\gamma_{i j}=\gamma \leq 1$ for all $i, j$. Moreover, $R \equiv \gamma^{-1}$ is the common radius of convergence of the generating functions

$$
P_{i j}(z) \equiv \sum_{n=0}^{\infty} p_{i j}(n) z^{n}, \quad i, j \geq 0
$$

In the transient case it is customary to make a further classification of random walks (and irreducible Markov chains in general) according to the limiting behaviour of $R^{n} p_{i j}(n)$ as $n \rightarrow \infty$. Indeed, the series $P_{i j}(R)$ either converge or diverge together. In the first case the random walk is called $R$-transient and in the latter $R$-recurrent. If $X$ is $R$-recurrent then either $\lim _{n \rightarrow \infty} R^{n} p_{i j}(n)=0$ for all $i, j$ or $\lim _{n \rightarrow \infty} R^{n} p_{i j}(n)>0$ for all $i, j$. In the first case $X$ is said to be $R$-null and in the latter $R$-positive. Evidently, a transient random walk is $R$-transient if $R=1$.

Our aim in the next subsections is to relate $\gamma$ to the support of the measure $\psi$ which goes with $X$, to obtain bounds for $\gamma$ and conditions for geometric ergodicity in terms of the 1-step transition probabilities of $X$, and to find criteria for deciding whether a transient random walk is $R$-transient, $R$-positive or $R$-null.

3.2. The aperiodic, transient random walk From (2.15) and Theorem (6.1) of Papangelou [23] (see also Kersting [18]) we know the following.

THEOREM 3.1. If the aperiodic random walk $X$ is transient, then its decay parameter $\gamma$ satisfies $\gamma=\eta(=\sup \operatorname{supp}(\psi))$.

Indeed, with (2.15) and (2.16) it is not difficult to verify directly from the representation formula (2.4) that $\gamma_{i j}=\eta$ for all $i, j$. Thus, finding representations and bounds for $\gamma$ in terms of the 1-step transition probabilities of $X$ amounts to finding representations and bounds for the largest limit point of the zeros of the random walk polynomials $\left\{Q_{j}(x)\right\}_{j}$ in terms of the parameters in the recurrence relation (2.5). Much is known about the latter problem. In particular, from van Doorn [6] we collect the following representations for $\eta$, which are in fact valid under milder conditions than we have assumed so far. 
LEMMA 3.1. Let $p_{i} q_{i+1}>0$ for all $i \geq 0$. Then the largest limit point $\eta$ of the (real) zeros of the polynomials $\left\{Q_{j}(x)\right\}_{j}$ satisfying the recurrence relation (2.5) can be represented as

(i) $\eta=\inf _{x} \sup _{j \geq 0}\left\{r_{j}+\chi_{j}^{-1} p_{j-1} q_{j}+\chi_{j+1}\right\}$, where $p_{-1} \equiv 0$ and $\chi \equiv\left(\chi_{0}, \chi_{1}, \ldots\right)$ is any sequence of positive numbers;

(ii) $\eta=\inf _{\beta} \sup _{j \geq 0} \frac{1}{2}\left\{r_{j}+r_{j+1}+\sqrt{\left(r_{j+1}-r_{j}\right)^{2}+4 \beta_{j+1}^{-1} p_{j} q_{j+1}}\right\}$, where $\beta$ is any chain sequence (that is, $\beta \equiv\left(\beta_{1}, \beta_{2}, \ldots\right)$ and each $\beta_{j}$ can be written as $\beta_{j}=$ $\left(1-g_{j-1}\right) g_{j}$ with $0 \leq g_{0}<1$ and $0<g_{j}<1$ for $\left.j>0\right)$;

(iii) $\eta=\sup _{\vartheta}\left\{\vartheta_{0} r_{0}+\sum_{k=1}^{\infty}\left(\vartheta_{k} r_{k}+2 \sqrt{\vartheta_{k-1} p_{k-1} \vartheta_{k} q_{k}}\right)\right\}$, where $\vartheta \equiv\left(\vartheta_{0}, \vartheta_{1}, \ldots\right)$ is any sequence of nonnegative numbers such that $\sum_{j} \vartheta_{j}=1$.

Combining Theorem 3.1 and Lemma 3.1 yields the following bounds for $\gamma$.

COROLLARY 3.1. If the aperiodic random walk $X$ is transient, then its decay parameter $\gamma$ satisfies the inequalities

(i) $\gamma \leq \sup _{j \geq 0}\left\{r_{j}+\sqrt{p_{j-1} q_{j}}+\sqrt{p_{j} q_{j+1}}\right\}$,

(ii) $\gamma \leq \sup _{j \geq 0} \frac{1}{2}\left\{r_{j}+r_{j+1}+\sqrt{\left(r_{j+1}-r_{j}\right)^{2}+16 p_{j} q_{j+1}}\right\}$,

(iii) $\gamma \geq \sup _{j \geq 0} \sup _{n \geq 1}\left\{r_{j}+\sum_{i=j+1}^{j+n-1}\left(r_{i}+2 \sqrt{p_{i-1} q_{i}}\right)\right\}$,

(iv) $\gamma \geq \sup _{j \geq 0} \frac{1}{2}\left\{r_{j}+r_{j+1}+\sqrt{\left(r_{j+1}-r_{j}\right)^{2}+4 p_{j} q_{j+1}}\right\}$.

PROOF. Choosing $\chi_{0}$ arbitrary and $\chi_{j}=\sqrt{p_{j-1} q_{j}}, j \geq 1$, the upper bound (i) follows from the first representation in Lemma 3.1. The upper bound (ii) is obtained from the second representation by taking $\beta_{j}=\frac{1}{4}\left(g_{j}=\frac{1}{2}\right)$ for all $j$. Choosing $\vartheta_{k}=1 / n$ for $k=0, j+1, \ldots, j+n-1$ and $\vartheta_{k}=0$ otherwise, the third representation gives the lower bound (iii), while the lower bound (iv) is obtained from this representation by choosing

$$
\begin{aligned}
& \vartheta_{j}=\frac{1}{2}-\frac{1}{2}\left(r_{j+1}-r_{j}\right) / \sqrt{\left(r_{j+1}-r_{j}\right)^{2}+4 p_{j} q_{j+1}} \\
& \vartheta_{j+1}=1-\vartheta_{j} \text { and } \vartheta_{k}=0 \text { for } k \neq j, j+1 .
\end{aligned}
$$

Concluding this subsection we give criteria for the transient random walk $X$ to be $R$-recurrent and $R$-positive (and hence $R$-transient and $R$-null).

THEOREM 3.2. Let the aperiodic random walk $X$ be transient with decay parameter $\gamma$ and let $R \equiv \gamma^{-1}$. Then the following are equivalent:

(i) $X$ is $R$-recurrent, 
(ii) $\sum_{j=0}^{\infty}\left(Q_{j}(\gamma) Q_{j+1}(\gamma) p_{j} \pi_{j}\right)^{-1}=\infty$,

(iii) $\int_{-1}^{1}(\gamma-x)^{-1} d \psi(x)=\infty$;

and the following are equivalent:

(i) $X$ is $R$-positive,

(ii) $\sum_{j=0}^{\infty} \pi_{j} Q_{j}^{2} \gamma<\infty$,

(iii) $\psi(\{\gamma\})>0$.

Proof. By Theorem 3.1, we have $\gamma=\eta$, so that $R=\eta^{-1}$. Applying the Derman-VereJones transformation of Appendix 2 to $X$, it follows that $R^{n} p_{i j}(n)=\left(Q_{i}(\eta) / Q_{j}(\eta)\right) \tilde{p}_{i j}(n)$, where $\left\{\tilde{p}_{i j}(n)\right\}$ are the transition probabilities of the random walk $\tilde{X}$ defined in (A2.1) which has $\tilde{q}_{0} \equiv 1-\tilde{p}_{0}-\tilde{r}_{0}=0$. Consequently, $X$ is $R$-recurrent ( $R$-positive) if and only if $\tilde{X}$ is recurrent (positive recurrent). The criteria given in the Theorems 2.1 and 2.3 (i), together with (A2.1), (A2.3) and (A2.4) now yield the required results.

3.3. The aperiodic, positive recurrent random walk When the aperiodic random walk $X$ is positive recurrent then $\psi(\{1\})>0$ by Theorem 2.3 (i). As a consequence, $\eta=1$, and hence $\psi(\{-1)\}=0$ by (2.18). By virtue of (2.3) and the representation formula (2.4), we can now write

$$
p_{i j}(n)-p_{i j}=\pi_{j} \int_{-1}^{1-} x^{n} Q_{i}(x) Q_{j}(x) d \psi(x) .
$$

By Lemma 2.2, we have $\xi=\inf \operatorname{supp}(\psi)$ and $\eta_{2}=\sup \{\operatorname{supp}(\psi) \backslash\{1\}\}$, and so, for all $i, j$,

$$
p_{i j}(n)-p_{i j}=\mathrm{O}\left(\rho^{n}\right) \text { as } n \rightarrow \infty, \text { with } \rho \equiv \max \left\{|\xi|, \eta_{2}\right\} .
$$

By looking at the case $i=j=0$, it becomes clear that the factor $\rho$ above cannot be improved, (that is, decreased) for all $i, j$ simultaneously, so the next result emerges.

THEOREM 3.3. If the aperiodic random walk $X$ is positive recurrent, then its decay parameter $\gamma$ satisfies $\gamma=\max \left\{|\xi|, \eta_{2}\right\}$.

REMARK. Examples can be constructed of positive recurrent random walks for which $Q_{i}(\gamma)=0$ for some $i \geq 0$, while $\psi$ has an isolated point mass at $\gamma$. Evidently, $\gamma_{i j}$ (and $\gamma_{j i}$ ) $<\gamma$ for all $j$ in such a case, which explains the phenomena described in Subsection 3.1.

In order to find representations and bounds for $\gamma$, we must now find representations and bounds for $\xi$ and $\eta_{2}$. As far as $\xi$ is concerned we can obtain results analogous to 
those for $\eta$ by a simple transformation of the results in Lemma 3.1 and its corollary. Indeed, it is readily seen that the polynomials $\left\{(-1)^{j} Q_{j}(-x)\right\}_{j}$ satisfy the recurrence (2.5) with $r_{j}$ replaced by $-r_{j}$. Hence, replacing $\eta$ by $-\xi$ and $r_{j}$ by $-r_{j}$ in Lemma 3.1 and its corollary gives us representations and bounds for $\xi$, see van Doorn [6]. As for $\eta_{2}$ the situation is more complicated, for we have at our disposal representations for the largest (or smallest) point in the support of a measure, given the parameters in the recurrence relation for the corresponding orthogonal polynomials, but not for the largest point but one. However, this problem can be circumvented by transforming the random walk polynomials $\left\{Q_{j}(x)\right\}_{j}$ into a set of polynomials $\left\{Q_{j}^{*}(x)\right\}_{j}$, such that the latter sequence is orthogonal with respect to a measure $\psi^{*}$ with the property $\eta^{*} \equiv \sup \operatorname{supp}\left(\psi^{*}\right)=\eta_{2}$. We can subsequently use techniques analogous to those of Lemma 3.1 and its corollary to obtain representations and bounds for $\eta^{*}$, and hence for $\eta_{2}$.

Concretely, we define

$$
Q_{j}^{*}(x) \equiv p_{j} \pi_{j}\left(Q_{j+1}(x)-Q_{j}(x)\right) /(x-1), \quad j \geq 0,
$$

and

$$
\psi^{*}([-1, x]) \equiv p_{0}^{-1} \int_{-1}^{x}(1-y) d \psi(y), \quad x \geq-1,
$$

so that $\xi^{*} \equiv \inf \operatorname{supp}\left(\psi^{*}\right)=\inf \operatorname{supp}(\psi)=\xi$ and $\eta^{*} \equiv \sup \operatorname{supp}\left(\psi^{*}\right)=\eta_{2}$. Then the sequence $\left\{Q_{j}^{*}(x)\right\}_{j}$ satisfies the recurrence relations

$$
\begin{aligned}
Q_{0}^{*}(x) & =1, \quad q_{1} Q_{1}^{*}(x)=x-1+p_{0}+q_{1} \\
x Q_{j}^{*}(x) & =p_{j} Q_{j-1}^{*}(x)+\left(1-p_{j}-q_{j+1}\right) Q_{j}^{*}(x)+q_{j+1} Q_{j+1}^{*}(x), j \geq 1,
\end{aligned}
$$

and is orthogonal with respect to $\psi^{*}$, namely,

$$
p_{0}\left(p_{j} \pi_{j}\right)^{-1} \int_{-1}^{1} Q_{i}^{*}(x) Q_{j}^{*}(x) d \psi^{*}(x)=\delta_{i j}
$$

This transformation is similar to the duality transformation used, for example, in van Doorn [5], [7] in the context of continuous-time birth-death processes, and a proof of the above results may be given on the basis of the corresponding results for continuous-time birth-death processes and the transformation described in (2.20); we shall not give the details. It should be noted that the polynomials $\left\{Q_{j}^{*}(x)\right\}_{j}$ of $(3.9)$ need not be random walk polynomials, so that the measure $\psi^{*}$ need not be a random walk measure. In particular, it can happen that $\xi^{*}+\eta^{*}<0$. In fact, $\left\{Q_{j}^{*}(x)\right\}_{j}$ constitutes a sequence of random walk polynomials if and only if $r_{j}^{*} \equiv 1-p_{j}-q_{j+1} \geq 0$ for all $j \geq 0$. Of course, $r_{j}^{*}<0$ for some $j$ need not prevent us from applying the representation formulas for $\eta^{*}$ that can be obtained from Lemma 3.1 by replacing $p_{j}$, $q_{j}$ and $r_{j}$ by $p_{j}^{*}=q_{j+1}, q_{j}^{*}=p_{j}$ and $r_{j}^{*}=1-p_{j}-q_{j+1}$, respectively. Again, we shall not write them down explicitly. 
3.4. Conditions for geometric ergodicity From the preceding sections we know that the aperiodic random walk $X$ is geometrically ergodic if $\eta<1$ (when $X$ is transient) or if $\max \left\{|\xi|, \eta_{2}\right\}<1$ (when $X$ is positive recurrent). It is easy to see from Theorem 2.3 (i) that if $X$ is null-recurrent, then $\eta=\eta_{2}=1$ and, as a consequence, $\gamma=1$, so that the random walk is not geometrically ergodic, as we had noticed already in Subsection 3.1. It follows that necessary and/or sufficient conditions for geometric ergodicity can be formulated on the basis of the representations and bounds for $\eta$ given in Lemma 3.1 and its corollary, and analogous results for $\xi$ and $\eta_{2}$. We shall not pursue this approach here, but rather give a criterion for geometric ergodicity in terms of the quantity $\tau$ that is defined in (2.12).

THEOREM 3.4. The aperiodic random walk $X$ is geometrically ergodic if and only if $\tau<1$.

PROOF. First suppose that the random walk is geometrically ergodic with decay parameter $\gamma<1$. Theorems 3.1 and 3.3 tell us that either $\gamma=\eta$ or $\gamma=\max \left\{|\xi|, \eta_{2}\right\}$. Since $\tau \leq \eta_{j}$ for all $j$ (see (2.11) and (2.12)) it follows that $\tau \leq \gamma<1$.

Next suppose $\tau<1$. Then $\sigma>-1$ by (2.16), and Lemma 2.2 implies that the random walk measure $\psi$ can only have isolated atoms outside the interval $[\sigma, \tau]$. Now if $\eta<1$, then the random walk is transient by Corollary 2.2, and hence $\gamma=\eta<1$ by Theorem 3.1. If, on the other hand, $\eta=1$, then $\psi$ has an isolated atom at 1 , and hence $\eta_{2}<1$. Also, $\psi$ cannot have an atom at -1 by $(2.18)$, so $|\xi|<1$ as well. Finally, the random walk is positive recurrent by Theorem 2.3 (i), and hence $\gamma=\max \left\{|\xi|, \eta_{2}\right\}<1$ by Theorem 3.3 .

It is well known (see Chihara [1, Theorem III.4.2]) that the value of $\tau$ depends only on the limiting behaviour as $j \rightarrow \infty$ of the parameters in the recurrence relation (2.5). This means that representations and bounds for $\tau$ usually lead to simpler and more powerful criteria for geometric ergodicity than representations and bounds for $\eta, \eta_{2}$ or $\xi$. We refer to the literature on orthogonal polynomials for various representations and bounds for $\tau$ (see for example, Chihara [1], [2] and van Doorn [4], [6]) and shall only mention the following powerful result (see [4]) and a corollary.

THEOREM 3.5. For any random walk, the parameter $\tau$ satisfies the inequalities

$$
\tau \leq \lim _{j \rightarrow \infty} \sup \left\{r_{j}+\sqrt{p_{j-1} q_{j}}+\sqrt{p_{j} q_{j+1}}\right\}
$$

and

$$
\tau \geq \lim _{n \rightarrow \infty} \sup \left\{\frac{1}{n} \sum_{j=1}^{n}\left(r_{j}+2 \sqrt{p_{j-1} q_{j}}\right)\right\} .
$$


The corollary pertains to the important case in which $p_{j}$ and $q_{j}$ tend to limits as $j \rightarrow \infty$, and may also be obtained from Blumenthal's Theorem (see Chihara [1]).

COROLLARY 3.6. If the aperiodic random walk $X$ has parameters satisfying $p_{j} \rightarrow p$, $q_{j} \rightarrow q$ and hence $r_{j} \rightarrow r \equiv 1-p-q$ as $j \rightarrow \infty$, then $\tau=1-(\sqrt{p}-\sqrt{q})^{2}$, so that $X$ is geometrically ergodic if and only if $p \neq q$.

3.5. Geometric ergodicity for periodic random walks It is clear that a periodic random walk $X$ has $p_{i j}(n)=0$ if $i+j$ and $n$ are of different parity. Indeed, since the random walk polynomials for $X$ satisfy $Q_{j}(x)=(-1)^{j} Q_{j}(-x)$, the representation formula (2.4) readily implies

$$
p_{i j}(n)=\pi_{j} \int_{0}^{1}\left((-1)^{i+j+n}+1\right) x^{n} Q_{i}(x) Q_{j}(x) d \psi(x), \quad n>0 .
$$

However, provided we let $n$ go to infinity in (3.1) and (3.2) through the even (odd) positive integers if $i+j$ is even (odd), we can maintain the definitions of $\gamma_{i j}$ and $\gamma$ in (3.2) and (3.3), and obtain the following result without difficulty.

THEOREM 3.7. If the periodic random walk $X$ is transient then $\gamma=\eta$; if $X$ is positive recurrent then $\gamma=\eta_{2}$.

Representations and bounds for the decay parameter of a periodic random walk may be obtained in the manner indicated in the Subsections 3.2 and 3.3. For example, the analogue of Corollary 3.1 for a periodic, transient random walk is very simple, namely,

$$
\sup _{j \geq 0} \sup _{n \geq 0}\left\{\frac{2}{n} \sum_{i=j+1}^{j+n-1} \sqrt{p_{i-1} q_{i}}\right\} \leq \gamma \leq \sup _{j \geq 0}\left\{\sqrt{p_{j-1} q_{j}}+\sqrt{p_{j} q_{j+1}}\right\},
$$

where $p_{-1} \equiv 0$, since the bounds (i) and (iii) in Corollary 3.1 are sharper than the bounds (ii) and (iv), respectively, when $r_{j} \equiv 0$.

Evidently, Theorem 3.4 retains its validity in the present context; so, as before, bounds for $\tau$ give us necessary or sufficient conditions for geometric ergodicity of a periodic random walk. In particular, the upper bound in Theorem 3.5 leads to the conclusion that

$$
\lim _{j \rightarrow \infty} \sup p_{j-1} q_{j}<\frac{1}{4}
$$

is sufficient for a periodic random walk to be geometrically ergodic. 


\section{Quasi-stationarity}

In this section we consider a random walk $X$ with the property $q_{0} \equiv 1-p_{0}-r_{0}>0$, so that -1 is an absorbing state. Also, we assume throughout that $\sum_{j}\left(p_{j} \pi_{j}\right)^{-1}=\infty$, so that eventual absorption at -1 is certain (see Theorem 2.2).

DEFINITION 4.1. An (honest) probability distribution $\left\{\alpha_{j}\right\}_{j=0}^{\infty}$ on the nonnegative integers is a quasi-stationary distribution for $X$ if the state probabilities $p_{j}(n) \equiv$ $\operatorname{Pr}\{X(n)=j\}$ of the random walk with initial distribution $p_{j}(0)=\alpha_{j}, j \geq 0$, satisfy

$$
p_{j}(n) /\left(1-p_{-1}(n)\right)=\alpha_{j}, \quad j \geq 0, n \geq 0 .
$$

In other words, a quasi-stationary distribution is an initial distribution such that the conditional probability of the process being in state $j$ at time $n$, given that no absorption has occurred by that time, is independent of $n$ for all $j$.

As a first step towards our goal of identifying all quasi-stationary distributions for $X$ we observe the following.

THEOREM 4.1. Let $X$ be a random walk with $q_{0}>0$ and $\sum_{j}\left(p_{j} \pi_{j}\right)^{-1}=\infty$, and $\left\{\alpha_{j}\right\}$ a probability distribution on the nonnegative integers. Then the following statements are equivalent:

(i) $\left\{\alpha_{j}\right\}$ constitutes a quasi-stationary distribution for $X$;

(ii) $\left\{\alpha_{j}\right\}$ solves the system

$$
\begin{aligned}
& \left(1-q_{0} \alpha_{0}\right) \alpha_{j}=p_{j-1} \alpha_{j-1}+r_{j} \alpha_{j}+q_{j+1} \alpha_{j+1}, \quad j \geq 1, \\
& \left(1-q_{0} \alpha_{0}\right) \alpha_{0}=r_{0} \alpha_{0}+q_{1} \alpha_{1}
\end{aligned}
$$

(iii) $\left\{\alpha_{j}\right\}$ solves the system

$$
\begin{aligned}
\sum_{i=0}^{\infty} \alpha_{i} p_{i j}(n) & =\alpha_{j}\left(1-q_{0} \alpha_{0}\right)^{n}, \quad j \geq 0 \\
\sum_{i=0}^{\infty} \alpha_{i} p_{i,-1}(n) & =1-\left(1-q_{0} \alpha_{0}\right)^{n} .
\end{aligned}
$$

PROOF. First suppose $\left\{\alpha_{j}\right\}$ is a quasi-stationary distribution. The state probabilities $p_{j}(n)=\sum_{i} \alpha_{i} p_{i j}(n)$ of the process with initial distribution $\left\{\alpha_{j}\right\}$ then satisfy $p_{j}(n)=$ $\alpha_{j}\left(1-p_{-1}(n)\right)$. But these state probabilities also satisfy the forward equations

$$
\begin{aligned}
p_{j}(n+1) & =p_{j-1} p_{j-1}(n)+r_{j} p_{j}(n)+q_{j+1} p_{j+1}(n), \quad j \geq 0, \\
p_{-1}(n+1) & =p_{-1}(n)+q_{0} p_{0}(n),
\end{aligned}
$$


where $p_{-1} \equiv 0$. It follows that $p_{j}(n+1)=\alpha_{j}\left(1-p_{-1}(n+1)\right)=\alpha_{j}\left(1-p_{-1}(n)-\right.$ $\left.q_{0} p_{0}(n)\right)=\alpha_{j}\left(1-q_{0} \alpha_{0}\right)\left(1-p_{-1}(n)\right)$. Substitution of these results in (4.4a) yields (4.2).

Next, let $\left\{\alpha_{j}\right\}$ be a solution of (4.2). Defining $\tilde{p}_{-1}(n)=1-\left(1-q_{0} \alpha_{0}\right)^{n}$ and $\tilde{p}_{j}(n)=\alpha_{j}\left(1-q_{0} \alpha_{0}\right)^{n}$, it is easily seen that $\left\{\tilde{p}_{j}(n)\right\}_{j=-1}^{\infty}, n \geq 0$, constitutes a sequence of probability distributions satisfying (4.4). Since $\tilde{p}_{-1}(0)=0$ and $\tilde{p}_{j}(0)=\alpha_{j}, j \geq 0$, we must have $\tilde{p}_{j}(n)=\sum_{i} \alpha_{i} p_{i j}(n)$, that is, $\left\{\alpha_{j}\right\}$ solves the system (4.3).

Finally, suppose $\left\{\alpha_{j}\right\}$ solves the system (4.3). Then, clearly, the state probabilities $p_{j}(n)=\sum_{i} \alpha_{i} p_{i j}(n), j \geq 0$, of the random walk with initial distribution $\left\{\alpha_{j}\right\}$ satisfy (4.1). That is, $\left\{\alpha_{j}\right\}$ constitutes a quasi-stationary distribution.

For notational convenience, we now introduce the functions

$$
a_{j}(x)=q_{0}^{-1} \pi_{j}(1-x) Q_{j}(x), \quad j \geq 0,
$$

where $\left\{Q_{j}(x)\right\}_{j}$ are the random walk polynomials of (2.5) associated with $X$ and $\left\{\pi_{j}\right\}$ the constants defined in (2.1). The next theorem, for the proof of which we use the results of Appendix 1, identifies all quasistationary distributions.

THEOREM 4.2. Let $X$ be a random walk with $q_{0}>0$ and $\sum_{j}\left(p_{j} \pi_{j}\right)^{-1}=\infty$. If $\eta=1$, then there exists no quasi-stationary distribution for $X$. If $\eta<1$, then there is a oneparameter family of quasi-stationary distributions, namely $\left\{\left\{a_{j}(x)\right\}_{j=0}^{\infty}, \eta \leq x<1\right\}$.

PROOF. From (4.2) and the recurrence relations (2.5) it is readily seen that any quasistationary distribution $\left\{\alpha_{j}\right\}$ must satisfy $\alpha_{j}=\alpha_{0} \pi_{j} Q_{j}\left(1-q_{0} \alpha_{0}\right)$, that is, $\alpha_{j}=a_{j}(x)$ for some $x$ and all $j \geq 0$. In order that $a_{j}(x) \geq 0$, we must have $\eta \leq x \leq 1$, as a consequence of (A1.1). But if $x=1$, then $a_{j}(x)=0$ for all $j$ and hence $\sum_{j} a_{j}(1)=0$. So we must have $\eta \leq x<1$. Hence there is no quasi-stationary distribution if $\eta=1$. In the case $\eta<1$ it remains to be determined for which values of $x, \eta \leq x<1$, one has $\sum_{j} a_{j}(x)=1$. To this end we observe from (4.5), (A1.3) and Lemma A1.1 that, for $x$ in the interval $[\eta, 1)$,

$$
\sum_{j=0}^{n} a_{j}(x)=q_{0}^{-1}(1-x) \sum_{j=0}^{n} \pi_{j} Q_{j}(x) \leq 1 .
$$

Since, by (A1.1), $Q_{j}(x)>0$ for $x \geq \eta$, it follows that $\pi_{j} Q_{j}(x) \rightarrow 0$ as $j \rightarrow \infty$ for $\eta \leq x<1$. Hence,

$$
p_{j} \pi_{j}\left(Q_{j+1}(x)-Q_{j}(x)\right)=q_{j+1} \pi_{j+1} Q_{j+1}(x)-p_{j} \pi_{j} Q_{j}(x) \rightarrow 0
$$

as $j \rightarrow \infty$ as well. Using (A1.3) again we conclude that $\sum_{j} a_{j}(x)=1$ for all $x$ in the interval $[\eta, 1)$. 
The theorem above and Theorems 3.1 and 3.7 give us the following interesting result.

COROLLARY 4.1. Quasi-stationary distributions for $X$ exist if and only if $X$ is geometrically ergodic.

A few comments on Theorem 4.2 and its corollary are in order. First, let $\eta<$ 1 , and suppose one chooses the quasi-stationary distribution $\left\{a_{j}(x)\right\}_{j}$ as the initial distribution for the random walk. Then, by Theorem 4.1, the state probabilities $p_{j}(n)=\sum_{i} a_{i}(x) p_{i j}(n)$ will satisfy

$$
p_{-1}(n)=1-x^{n} \quad \text { and } \quad p_{j}(n)=a_{j}(x) x^{n}, \quad j \geq 0 .
$$

Secondly, we note that Theorem 4.2 and its corollary together with some of our findings in Section 3 concretize, generalize and supplement the recent results of Ferrari, Martínez and Picco [11].

Finally, solving (4.2) for a probability distribution is equivalent to finding a nonnegative, nonzero, summable solution $\boldsymbol{y} \equiv\left(y_{0}, y_{1}, \ldots\right)$ to the eigenvector problem

$$
x \boldsymbol{y}=\boldsymbol{y} P,
$$

where $P$ is the matrix of 1 -step transition probabilities given in (1.1). Indeed, for any nonnegative, nonzero, summable solution $y$ of (4.7), normalized such that $\sum_{j} y_{j}=$ 1 , one must have $y_{0}=q_{0}^{-1}(1-x)$, as can be seen by post-multiplying (4.7) by the vector consisting of 1 's. It subsequently follows that a solution to (4.7) must satisfy $y_{j}=a_{j}(x)$. From Theorem 4.2 , we therefore conclude that there exists a nonnegative, nonzero, summable solution to (4.7) if and only if $\eta \leq x<1$. In the more general context of infinite Markov chains, the problem of finding all quasistationary distributions again amounts to solving the eigenvector problem (4.7) with $\eta \leq x<1$, where $\eta$ is the decay parameter of the chain. Then, however, it is not known whether suitable eigenvectors exist, unless $x=\eta$ and the chain is $R$-positive, see Vere-Jones [28], Seneta and Vere-Jones [26], Kijima [19]. Recent advances for continuous time Markov chains (Ferrari, Kesten, Martínez and Picco [10]) suggest, however, that Corollary 4.1 is valid in much greater generality.

Concluding this subsection, the next theorem gives an interesting relationship between the members of the family of quasi-stationary distributions, to the effect that if $\eta \leq y<x<1$, then the quasi-stationary distribution $\left\{a_{j}(x)\right\}_{j}$ is strictly stochastically larger than the quasi-stationary distribution $\left\{a_{j}(y)\right\}_{j}$ in the sense of monotone likelihood-ratio ordering (see, for example, Whitt [30]).

THEOREM 4.3. Let $X$ be a random walk with $q_{0}>0$ and $\sum_{j}\left(p_{j} \pi_{j}\right)^{-1}=\infty$ and suppose $\eta \leq y<x<1$. Then $a_{0}(x)<a_{0}(y)$ and

$$
a_{j+1}(y) / a_{j}(y)<a_{j+1}(x) / a_{j}(x), \quad j \geq 0 .
$$


PRoOF. If $\eta \leq y<x<1$, then $a_{0}(x)=q_{0}^{-1}(1-x)<q_{0}^{-1}(1-y)=a_{0}(y)$. Moreover, exploiting the recurrence relations (2.5) and noting that $q_{n+1} \pi_{n+1}=p_{n} \pi_{n}$ we easily obtain

$$
(x-y) \sum_{k=0}^{j} \pi_{k} Q_{k}(x) Q_{k}(y)=p_{j} \pi_{j}\left(Q_{j}(y) Q_{j+1}(x)-Q_{j}(x) Q_{j+1}(y)\right),
$$

which is the Christoffel-Darboux identity (see Chihara [1]). Hence, for $\eta \leq y<x<$ 1 ,

$$
Q_{j}(y) Q_{j+1}(x)>Q_{j}(x) Q_{j+1}(y),
$$

since $Q_{k}(x) Q_{k}(y)>0$ by (A1.1). The validity of (4.8) follows with (4.5).

\section{Examples}

We first consider the random walk with parameters

$$
p_{j}=p, \quad q_{j}=q \equiv 1-p, \quad r_{j}=0, \quad j \geq 0 .
$$

Karlin and McGregor [15] have shown that the associated random walk polynomials can be expressed as

$$
Q_{j}(x)=(q / p)^{j / 2} U_{j}(x / \sqrt{4 p q}),
$$

where $U_{j}(\cdot)$ is a Tchebichef polynomial of the second kind (see, for example, Chihara [1]) while the corresponding measure consists of the density

$$
\psi^{\prime}(x)=(2 \pi p q)^{-1} \sqrt{4 p q-x^{2}}
$$

over the interval $-\sqrt{4 p q}<x<\sqrt{4 p q}$. It follows immediately that for $p \neq \frac{1}{2}$, the random walk is geometrically ergodic with decay parameter $\sqrt{4 p q}$.

Assuming $p<\frac{1}{2}$, absorption at -1 is easily seen to be ergodic. So, by Theorem 4.2, there exists a family of quasi-stationary distributions $\left\{\left\{a_{j}(x)\right\}_{j}, \sqrt{4 p q} \leq x<1\right\}$, where

$$
a_{j}(x)=q^{-1}(p / q)^{j / 2}(1-x) U_{j}(x / \sqrt{4 p q}), j=0,1, \ldots .
$$

In particular, since $U_{j}(1)=j+1$, the extremal quasi-stationary distribution corresponding to $x=\eta=\sqrt{4 p q}$ is given by

$$
a_{j}(\eta)=(1-\sqrt{p / q})^{2}(j+1)(p / q)^{j / 2}, j=0,1, \ldots .
$$

Not surprisingly, this quasi-stationary distribution is identical to that of the birth-death process with constant birth rates $\lambda$ and death rates $\mu$ such that $\lambda / \mu=p / q$ (see for example, van Doorn [7]). 
We next look at the generalization of (5.1) where $p_{j}=p, q_{j}=q \equiv 1-p$ and $r_{j}=0$ for $j \geq 1$, but $p_{0}$ and $r_{0}$ are arbitrary (and $q_{0}=1-r_{0}-p_{0} \geq 0$ ). Writing the associated random walk polynomials $Q_{j}(x)$ as

$$
Q_{j}(x)=(q / p)^{j / 2} P_{j}(x / \sqrt{4 p q}),
$$

the polynomials $\left\{P_{j}(x)\right)_{j}$ are readily seen to satisfy the recurrence relations

$$
\begin{aligned}
& P_{0}(x)=1, \quad P_{1}(x)=2\left(p / p_{0}\right) x-\left(r_{0} / p_{0}\right) \sqrt{p / q} \\
& 2 x P_{j}(x)=P_{j-1}(x)+P_{j+1}(x), \quad j \geq 1 .
\end{aligned}
$$

Chihara [1, page 204], referring to earlier work of Geronimus, subsequently gives us the representation

$$
P_{j}(x)=a T_{j}(x)+(a-1) U_{j-2}(x)-b U_{j-1}(x),
$$

where $\left\{T_{j}(x)\right\}_{j}$ and $\left\{U_{j}(x)\right\}_{j}$ are the Tchebichef polynomials of the first and second kind, respectively, and

$$
a \equiv 2 p / p_{0} \text { and } b \equiv\left(r_{0} / p_{0}\right) \sqrt{p / q}
$$

For the special cases $b=0$ (that is, $r_{0}=0$ ), $a=1$ (that is, $p_{0}=2 p$ ) and $a=2$, (that is, $p_{0}=p$ ), Chihara [1, pages 204-205] gives us the corresponding measures, which generalizes the results of Karlin and McGregor [15]. On the other hand, Karlin and McGregor [15] describe a procedure to calculate the measure in the general case, see also Sansigre and Valent [25].

Looking more closely into the case $p_{0}=p(a=2)$, Chihara's [1] results readily reveal that $\eta=\sup \operatorname{supp}(\psi)$ is given by

$$
\eta= \begin{cases}2 \sqrt{p q} & \text { if } r_{0} \leq \sqrt{p q} \\ r_{0}+r_{0}^{-1} p q & \text { if } r_{0}>\sqrt{p q}\end{cases}
$$

That is, $\eta$ as a function of $r_{0}$ is constant as long as $r_{0} \leq \sqrt{p q}$, but increases to 1 as $r_{0}$ increases from $\sqrt{p q}$ to its maximum value $q$. Of course, $q_{0}>0$ as long as $r_{0}<q$. If $p<\frac{1}{2}$ and $r_{0}<q$, then absorption at -1 is easily seen to be ergodic, so that an infinite family of quasi-stationary distributions exists. Exploiting the facts that $T_{j}(1)=1$ and $U_{j}(1)=j+1$, the preceding results readily reveal that, when $r_{0} \leq \sqrt{p q}$, the extremal quasi-stationary distribution corresponding to $x=\eta=2 \sqrt{p q}$ is given by

$$
a_{j}(\eta)=q q_{0}^{-1}(1-\sqrt{p / q})^{2}\left(1+\left(1-r_{0} / \sqrt{p q}\right) j\right)(p / q)^{j / 2}, j=0,1, \ldots
$$




\section{Appendix 1: A lemma involving random walk polynomials}

Let $\left\{Q_{j}(x)\right\}$ be a sequence of random walk polynomials as defined in Section 2.2. Since the coefficient of $x^{j}$ in $Q_{j}(x)$ is positive, it is clear from the definition of $\eta \equiv \eta_{1}$ in $(2.10)$ that

$$
x \geq \eta \Leftrightarrow Q_{j}(x)>0 \text { for all } j \geq 0 .
$$

We can obtain more detailed information about the sequence $\left\{Q_{j}(x)\right\}_{j}$ when it corresponds with a random walk with $q_{0} \equiv 1-p_{0}-r_{0}>0$ for which absorption is certain (see Theorem 2.2). To this end we first note that the recurrence relations (2.5) can be written in the form

$$
\begin{aligned}
& (x-1) \pi_{0} Q_{0}(x)=p_{0} \pi_{0}\left(Q_{1}(x)-Q_{0}(x)\right)-q_{0} \\
& (x-1) \pi_{j} Q_{j}(x)=p_{j} \pi_{j}\left(Q_{j+1}(x)-Q_{j}(x)\right)-p_{j-1} \pi_{j-1}\left(Q_{j}(x)-Q_{j-1}(x)\right), j \geq 1,
\end{aligned}
$$

since $p_{j} \pi_{j}=q_{j+1} \pi_{j+1}$. Consequently,

$$
p_{j} \pi_{j}\left(Q_{j+1}(x)-Q_{j}(x)\right)=q_{0}+(x-1) \sum_{k=0}^{j} \pi_{k} Q_{k}(x), j \geq 0 .
$$

We can now establish the announced generalization of (A1.1), which is of importance in the analysis of Section 4.

LEMMA A1.1. Let $q_{0}>0$ and $\sum_{j}\left(p_{j} \pi_{j}\right)^{-1}=\infty$. If $x \geq \eta$ then $Q_{j+1}(x)>Q_{j}(x)$ for all $j>0$.

ProOF. By virtue of (A1.3), the conclusion of the lemma is obviously true for $x \geq 1$, since then, by (A1.1) and Lemma 2.1, $Q_{j}(x)>0$ for all $j$. (So in this case $\left\{Q_{j}(x)\right\}_{j}$ is monotonically increasing also if $\sum_{j}\left(p_{j} \pi_{j}\right)^{-1}<\infty$.) Therefore, let us assume $\eta \leq x<1$. From (A1.1) and (A1.3) it then follows that the sequence $\left\{a_{j}\right\}_{j=0}^{\infty}$, where $a_{j} \equiv p_{j} \pi_{j}\left(Q_{j+1}(x)-Q_{j}(x)\right)$, is strictly decreasing. Considering that

$$
Q_{j+1}(x)=1+\sum_{k=0}^{j}\left(p_{k} \pi_{k}\right)^{-1} a_{k}, j \geq 0
$$

the assumption $a_{j}<0$ for some $j$ leads to a contradiction, since $Q_{j+1}(x)>0$ while $\sum_{k=0}^{j}\left(p_{k} \pi_{k}\right)^{-1}$ tends to $+\infty$ as $j \rightarrow \infty$. Consequently, $a_{j} \geq 0$ for all $j$, and hence $a_{j}>0$, that is, $Q_{j+1}(x)>Q_{j}(x)$, for all $j$. 


\section{Appendix 2: The Derman-Vere-Jones transformation}

Let $X$ denote an arbitrary but fixed random walk, $\left\{Q_{j}(x)\right\}_{j}$ the corresponding sequence of random walk polynomials, and $\psi$ the corresponding random walk measure. As usual, we let $q_{0} \equiv 1-p_{0}-r_{0}$ and allow $0 \leq q_{0}<1$. We recall that

$$
-1 \leq \xi=\inf \operatorname{supp}(\psi)<\sup \operatorname{supp}(\psi)=\eta \leq 1
$$

and $\xi+\eta \geq 0$. We will describe a mapping by which the random walk $X$ is transformed into a random walk $\tilde{X}$ with $\tilde{q}_{0}=0$ and $\tilde{\eta}=1$. The transformation was introduced, in a more general context, by Derman [3] and generalized by Vere-Jones [28], and therefore we will refer to it as the Derman-Vere-Jones transformation.

Concretely, the Derman-Vere-Jones transformation maps the set of random walk parameters $\left\{p_{j}, q_{j+1}, r_{j}\right\}_{j=0}^{\infty}$ into the set of random walk parameters $\left\{\tilde{p}_{j}, \tilde{q}_{j+1}, \tilde{r}_{j}\right\}_{j=0}^{\infty}$ defined by

$$
\left.\begin{array}{l}
\tilde{p}_{j} \equiv \eta^{-1}\left(Q_{j+1}(\eta) / Q_{j}(\eta)\right) p_{j} \\
\tilde{r}_{j} \equiv \eta^{-1} r_{j} \\
\tilde{q}_{j+1} \equiv \eta^{-1}\left(Q_{j}(\eta) / Q_{j+1}(\eta)\right) q_{j+1} .
\end{array}\right\}
$$

Indeed, by (A1.1) we have $\tilde{p}_{j}>0, \tilde{q}_{j+1}>0$, and by the recurrence relation (2.5) it follows that $\tilde{q}_{0}=1-\tilde{p}_{0}-\tilde{r}_{0}=0$ and $\tilde{p}_{j}+\tilde{q}_{j}+\tilde{r}_{j}=1$ for $j \geq 1$. The corresponding sequence of random walk polynomials $\left\{\tilde{Q}_{j}(x)\right\}_{j}$ is readily verified to satisfy

$$
\tilde{Q}_{j}(x)=Q_{j}(x \eta) / Q_{j}(\eta)
$$

and it follows that

$$
\tilde{\pi}_{j} \int_{-1}^{1} \tilde{Q}_{i}(x) \tilde{Q}_{j}(x) d \tilde{\psi}(x)=\delta_{i j}, \quad i, j \geq 0
$$

where

$$
\tilde{\pi}_{j}=\pi_{j} Q_{j}^{2}(\eta)
$$

and $\tilde{\psi}$, defined by

$$
\tilde{\psi}([-1, x])=\psi([-\eta, x \eta]),-1 \leq x \leq 1,
$$

is the corresponding random walk measure. It is evident that $\tilde{\eta} \equiv \sup \operatorname{supp}(\tilde{\psi})=1$, while $\tilde{\xi} \equiv \inf \operatorname{supp}(\tilde{\psi})=\xi \eta^{-1} \geq-1$. The $n$-step transition probabilities $\tilde{p}_{i j}(n)$, $i, j \geq 0$, associated with $\tilde{X}$ can now be expressed as

$$
\begin{aligned}
\tilde{p}_{i j}(n) & =\tilde{\pi}_{j} \int_{-1}^{1} x^{n} \tilde{Q}_{i}(x) \tilde{Q}_{j}(x) d \tilde{\psi}(x) \\
& =\pi_{j}\left(Q_{j}(\eta) / Q_{i}(\eta)\right) \eta^{-n} \int_{-1}^{1}(x \eta)^{n} Q_{i}(x \eta) Q_{j}(x \eta) d \psi(x \eta) \\
& =\eta^{-n}\left(Q_{j}(\eta) / Q_{i}(\eta)\right) p_{i j}(n),
\end{aligned}
$$


so that the transition probabilities of $X$ satisfy

$$
p_{i j}(n)=\eta^{n}\left(Q_{i}(\eta) / Q_{j}(\eta)\right) \tilde{p}_{i j}(n), i, j \geq 0
$$

Thus the Derman-Vere-Jones transformation enables us to analyse a general random walk through a random walk with $q_{0}=0$ and $\eta=1$, a feature which is employed in the proof of Theorem 3.2 .

\section{References}

[1] T. S. Chihara, An introduction to orthogonal polynomials (Gordon and Breach, New York, 1978).

[2] T. S. Chihara, 'Spectral properties of orthogonal polynomials on unbounded sets', Trans. Amer. Math. Soc. 270 (1982) 623-639.

[3] C. Derman, 'A solution to a set of fundamental equations in Markov chains', Proc. Amer. Math. Soc. 5 (1954) 332-334.

[4] E. A. van Doorn, 'On oscillation properties and the interval of orthogonality of orthogonal polynomials', SIAM J. Math. Anal. 15 (1984) 1031-1042.

[5] E. A. van Doom, 'Conditions for exponential ergodicity and bounds for the decay parameter of a birth-death process', Adv. Appl. Probab. 17 (1985) 514-530.

[6] E. A. van Doorn, 'Representations and bounds for zeros of orthogonal polynomials and eigenvalues of sign-symmetric tri-diagonal matrices', J. Approx. Th. 51 (1987) 254-266.

[7] E. A. van Doorn, 'Quasi-stationary distributions and convergence to quasi-stationarity of birthdeath processes', Adv. Appl. Probab. 23 (1991) 683-700.

[8] E. A. van Doom and P. Schrijner, 'Random walk polynomials and random walk measures', $J$. Comput. Appl. Math. 49 (1993) 289-296.

[9] E. A. van Doorn and P. Schrijner, 'Ratio limits and limiting conditional distributions for discretetime birth-death processes', J. Math. Anal. Appl. 190 (1995) 263-284.

[10] P. A. Ferrari, H. Kesten, S. Martínez and P. Picco, 'Existence of quasistationary distributions. A renewal dynamical approach', Ann. Probab., to appear.

[11] P. A. Ferrari, S. Martínez and P. Picco, 'Existence of non trivial quasi stationary distributions in the birth and death chain', Adv. Appl. Probab. 24 (1992) 795-813.

[12] T. E. Harris, 'First passage and recurrence distributions', Trans. Amer. Math. Soc. 73 (1952) $471-486$.

[13] S. Karlin and J. L. McGregor, 'The differential equations of birth-and-death processes, and the Stieltjes moment problem', Trans. Amer. Math. Soc. 85 (1957) 489-546.

[14] S. Karlin and J. L. McGregor, 'The classification of birth and death processes', Trans. Amer. Math. Soc. 86 (1957), 366-400.

[15] S. Karlin and J. L. McGregor, 'Random walks', Illinois J. Math. 3 (1959), 66-81.

[16] D. G. Kendall, 'Unitary dilations of Markov transition operators and the corresponding integral representation for transition probability matrices', pp. 139-161, in: Surveys in probability and statistics - The Harold Cramer volume, U. Grenander, ed. (Almquist and Wiksell, Stockholm, 1959).

[17] J. T. Kent and N. T. Longford, 'An eigenvalue decomposition for first hitting times in random walks', Z. Wahrscheinlichkeitstheorie verw. Gebiete 63 (1983) 71-84.

[18] G. Kersting, 'Strong ratio limit property and R-recurrence of reversible Markov chains', Z. Wahrscheinlichkeitstheorie verw. Gebiete 30 (1974) 343-356. 
[19] M. Kijima, 'On the existence of quasi-stationary distributions in denumerable R-transient Markov chains', J. Appl. Probab. 29 (1992) 21-36; Correction, 30 (1993) 496.

[20] M. Kijima, 'Quasi-stationary distributions of single-server phase-type queues', Math. Oper. Res. 18 (1993) 423-437.

[21] J. F. C. Kingman, 'The exponential decay of Markov transition probabilities', Proc. London Math. Soc. 13 (1963) 337-358.

[22] M. G. Nair and P. K. Pollett, 'On the relationship between $\mu$-invariant measures and quasistationary distributions for continuous-time Markov chains', Adv. Appl. Probab. 25 (1993) 82-102.

[23] F. Papangelou, 'Strong ratio limits, R-recurrence and mixing properties of discrete parameter Markov processes', Z. Wahrscheinlichkeitstheorie verw. Gebrete 8 (1967) 259-297.

[24] P. K. Pollett, 'Recent advances in the theory and application of quasistationary distributions', pp. 477-486, in Stochastic models in engineering, technology and management, eds. S. Osaki and D. N. P. Murthy, (World Scientific, Singapore, 1993).

[25] G. Sansigre and G. Valent, "A large family of semi-classical polynomials: the perturbed Tchebichev', J. Comput. Appl. Math. to appear.

[26] E. Seneta and D. Vere-Jones, 'On quasi-stationary distributions in discrete-time Markov chains with a denumerable infinity of states', J. Appl. Probab. 3 (1966) 403-434.

[27] J. A. Shohat and J. D. Tamarkin, The problem of moments. Mathematical Surveys No I, rev. edition (American Mathematical Society, Providence, R. I., 1963).

[28] D. Vere-Jones, 'Geometric ergodicity in denumerable Markov chains', Quart. J. Math. Oxford (2) 13 (1962) 7-28.

[29] T. A. Whitehurst, On random walks and orthogonal polynomials. Ph.D. Thesis (Indiana University, Bloomington, 1978).

[30] W. W. Whitt, 'The renewal-process stationary-excess operator', J. Appl. Probab. 22 (1985) 156167. 